\title{
Unbinding of the streptavidin-biotin complex by atomic force microscopy: A hybrid simulation study
}

\author{
Jian Zhou, ${ }^{\text {a) }}$ Luzheng Zhang, and Yongsheng Leng \\ Department of Chemical Engineering, University of Washington, Seattle, Washington 98195 \\ Heng-Kwong Tsao \\ Department of Chemical Engineering, National Central University, Chung-li 320, Taiwan \\ Yu-Jane Sheng \\ Department of Chemical Engineering, National Taiwan University, Taipei 106, Taiwan \\ Shaoyi Jiang ${ }^{\text {b) }}$ \\ Department of Chemical Engineering, University of Washington, Seattle, Washington 98195
}

(Received 13 June 2006; accepted 25 July 2006; published online 13 September 2006)

\begin{abstract}
A hybrid molecular simulation technique, which combines molecular dynamics and continuum mechanics, was used to study the single-molecule unbinding force of a streptavidin-biotin complex. The hybrid method enables atomistic simulations of unbinding events at the millisecond time scale of atomic force microscopy (AFM) experiments. The logarithmic relationship between the unbinding force of the streptavidin-biotin complex and the loading rate (the product of cantilever spring constant and pulling velocity) in AFM experiments was confirmed by hybrid simulations. The unbinding forces, cantilever and tip positions, locations of energy barriers, and unbinding pathway were analyzed. Hybrid simulation results from this work not only interpret unbinding AFM experiments but also provide detailed molecular information not available in AFM experiments.

(c) 2006 American Institute of Physics. [DOI: 10.1063/1.2337629]
\end{abstract}

\section{INTRODUCTION}

In many physiological processes, cell adhesion plays an essential role in diverse biological phenomena including inflammation and cancer metastasis, where the binding and unbinding of receptor-ligand pairs are extensively involved. The receptor-ligand interactions (e.g., in the streptavidinbiotin complex) are also very important in applications such as biosensors and biomaterials. Thus, it is essential to study the receptor-ligand interactions.

In the past decade, there are increasing interests in determining the single-molecule forces of receptor-ligand complexes $^{1-4}$ by dynamic force spectroscopy (DFS). DFS experimental tools include atomic force microscope ${ }^{5-9,9-12}$ (AFM), biomembrane force probe ${ }^{13}$ (BFP), and optical tweezer $^{14}$ (OT). Among these methods, AFM is the most widely used technique. To determine the unbinding force by AFM, a receptor is anchored to a surface while a ligand is covalently linked to an AFM tip. The force probe coated with the ligand is brought into contact with the substrate covered with the receptor (binding). On retraction of the tip from the surface (unbinding), the spring force is recorded as a function of cantilever position (or time). In such experiments, the unbinding (or rupture) force is defined as the largest spring force applied during the process.

The streptavidin (or avidin)-biotin system is one of the

\footnotetext{
${ }^{\text {a) }}$ Present address: School of Chemical and Energy Engineering, South China University of Technology, GuangZhou, GuangDong Province, 510640, People's Republic of China.

b) Author to whom correspondence should be addressed. FAX: (206)-685-

3451. Electronic mail: sjiang@u.washington.edu
}

strongest noncovalent interactions in nature. It is also a model system for studying receptor-ligand interactions. Experimentally, Lee et al..$^{5}$ demonstrated the capability of AFM to measure discrete and biologically specific rupture forces of this system at the single-molecule level. They found that surfaces functionalized with biotin and streptavidin exhibited adhesive forces three to eight times greater than nonspecific interactions observed between blocked streptavidin and biotinylated surfaces. The obtained unbinding forces for streptavidin-biotin are between 300 and $400 \mathrm{pN}$ for cantilevers with different spring constants. Gaub and co-workers ${ }^{6,7}$ showed the direct measurements of the rupture of individual biologically specific bonds; the unbinding forces are 160 and $257 \mathrm{pN}$ for avidin-biotin and streptavidin-biotin, respectively. The unbinding forces of the streptavidin (or avidin)biotin system were further investigated with statistical analysis $^{8-10}$ and discrete methods. ${ }^{11,12}$ Patel et al. ${ }^{15}$ highlighted the influence of molecular architecture on the kinetic stability of this system.

Evans and Ritchie ${ }^{16}$ studied the strength of weak noncovalent bonds in liquids. Although the streptavidin-biotin system is among the strongest noncovalent linkages in biology, these bonds can appear strong or weak in dynamic measurements, depending on how fast they are loaded. They ${ }^{16}$ used Kramers' theory and derived a formula to predict the dependence of unbinding forces on loading rates. When their predictions were applied to the range of loading rates attainable for AFM, a logarithmic dependence of unbinding force on loading rates was observed. They ${ }^{16}$ further predicted that, for a range of loading rates covering 12 orders of magnitude, the 
rupture force of an avidin-biotin complex could not be fitted with a single exponential. These predictions were confirmed by their BFP experiments ${ }^{13}$ later. The same dependence was also found by others from AFM experiments. ${ }^{9,12}$

For experimental studies, one difficulty is how to ensure that a particular force measurement actually deals with a single interacting pair between the tip and the substrate. Another difficulty is to differentiate specific interactions from nonspecific interactions. When the tip approaches the sample, the adsorbed protein at the interface could experience mild denaturation. It is hard to determine if the force curves obtained from AFM experiments truly correspond to the unbinding events of a native protein or the stretching of a partially denatured protein. The unbinding and mechanical properties of the receptor-ligand complex under an external force also largely depend on the orientation of the complex and its degrees of freedom. In addition to these difficulties, the atomic details of the unbinding process are not available from AFM experiments. Molecular simulations are well suited for studying truly single-molecule interactions, revealing the molecular-level unbinding mechanism and providing detailed information during the unbinding process. Simulation results will complement those from experiments very well.

Grubmuller et al. ${ }^{17}$ pioneered the molecular simulation study of the unbinding of a streptavidin-biotin complex. A streptavidin monomer with a bound biotin was first equilibrated in a bath of water molecules. Force was then exerted on the biotin to unbind the complex. However, the unbinding process was simulated at very fast cantilever pulling velocities for $1 \mathrm{~ns}$ in their molecular dynamics (MD) study. Furthermore, a relatively stiff spring (spring constant of $2.8 \mathrm{~N} / \mathrm{m}$ ) was used, which is much stiffer than that used in AFM experiments (spring constant of $\sim 0.05 \mathrm{~N} / \mathrm{m}$ ). Very fast pulling velocities and the stiff spring constant used lead to very high loading rates of $4.2-112 \mathrm{~N} / \mathrm{s}$. A mathematical procedure was also used to process their raw data; they smoothed the force profile with a Gaussian distribution. The unbinding force obtained in this way was between $300-650 \mathrm{pN}$. Izrailev et al. ${ }^{18}$ simulated the unbinding of an avidin-biotin complex in a continuum solvent with a slowly stiffening cantilever $(0-0.414 \mathrm{~N} / \mathrm{m})$ at very fast cantilever pulling velocities. The range of their loading rates is $1.3-83 \mathrm{~N} / \mathrm{s}$. The obtained unbinding force for the avidinbiotin system was $450 \mathrm{pN}$. For these studies ${ }^{17,18}$ using conventional MD simulation methods, the loading rates (or cantilever pulling velocities) are many orders of magnitude higher than those of AFM experiments. As suggested by Grubmuller et al. ${ }^{17}$ and discussed by Izrailev et al. ${ }^{18}$ frictional effects are expected to contribute significantly to the unbinding force of receptor-ligand complexes at pulling velocities above $1.0 \mathrm{~m} / \mathrm{s}$ (friction region). In AFM experiments, activated processes dominate at slower velocities of $\sim 1.0 \mu \mathrm{m} / \mathrm{s}$ (activated region), for which frictional forces become negligible. Thus, it is hard to directly compare the unbinding forces obtained from MD simulations ${ }^{17,18}$ at very fast pulling velocities $(\sim 1.0 \mathrm{~m} / \mathrm{s})$ with those from AFM experiments at slow pulling velocities $(\sim 1.0 \mu \mathrm{m} / \mathrm{s})$. By considering activated unbinding and frictional forces, Heymann and Grubmuller ${ }^{19}$ used a simple model to extrapolate the simulated unbinding forces to the millisecond time regime of single-molecule AFM unbinding experiments for the AN02/ DNP-hapten system. However, their extrapolation could only be obtained for a simplified binding interaction potential and based on the assumption that there is only one single energy barrier along the unbinding trajectory. Galligan et al. ${ }^{20}$ adopted a combination of traditional reaction coordinate mapping and Brownian dynamics to simulate the dynamic strength of streptavidin-biotin interactions. They ${ }^{20}$ also observed the logarithmic dependence of rupture forces on loading rates.

In this work, a hybrid molecular simulation technique, which combines continuum mechanics and molecular dynamics, was used to simulate the unbinding of a streptavidinbiotin complex ${ }^{21}$ at the experimental AFM time scale. Previous experiments ${ }^{9,12,13}$ have shown that there is a logarithmic relationship between unbinding forces and loading rates. Thus, the objectives of this work are to simulate the singlemolecule unbinding process at the AFM experimental time scale, to verify the loading-rate-dependent unbinding forces found in AFM experiments and to explore the unbinding pathway and locations of energy barriers of the streptavidinbiotin complex at the molecular level.

\section{SIMULATION METHODOLOGY}

Previously, a hybrid simulation method ${ }^{22}$ was developed to study the adhesion and friction of self-assembled monolayers on $\mathrm{Au}(111){ }^{22-24}$ Since the mass of an atom is many orders of magnitude different from that of an AFM tip, their characteristic vibration frequencies are very different. Therefore, the atomic motion and the tip motion can be decoupled. For hybrid simulations, the integration of the dynamic equations of motion for the AFM tip and the complex system can be done separately, i.e., the equations of motion for the tip is integrated with a comparably larger time step ( $\mu \mathrm{s})$ and then a fast relaxation of the atomic complex by MD simulations with a smaller time step (fs) is followed. Quasistatic assumption is adopted, during the tip motion, the molecular system should be well preserved in thermal fluctuation. In this way, we can simulate the streptavidin-biotin unbinding process by AFM at pulling velocities or loading rates close to AFM unbinding experiments.

In simulations of an unbinding process, the AFM tip is dragged by a support through a spring $\left(k_{x}, k_{y}, k_{z}\right)$ at a velocity of $v$. The differential equations of motion of the AFM tip are governed by

$$
\begin{aligned}
& M \ddot{x}_{t}=k_{x}\left(x_{M}-x_{t}\right)+W_{x}\left(x_{t}, y_{t}, z_{t} ; X(t)\right), \\
& M \ddot{y}_{t}=k_{y}\left(y_{M}-y_{t}\right)+W_{y}\left(x_{t}, y_{t}, z_{t} ; X(t)\right), \\
& M \ddot{z}_{t}=k_{z}\left(z_{M}-z_{t}\right)+W_{z}\left(x_{t}, y_{t}, z_{t} ; X(t)\right),
\end{aligned}
$$

where the forces contributed by the receptor and streptavidin 
are denoted by $W_{x}, W_{y}$, and $W_{z}$ in the $x, y$, and $z$ directions. These forces depend not only on the tip position $\left(x_{t}, y_{t}, z_{t}\right)$ but also on the configuration of the receptor-ligand complex studied $X(t)$ at time $t . M$ is the effective mass ${ }^{25}$ of the tip, $M=10^{-11} \mathrm{~kg}$. The position of the support is $\left(x_{M}, y_{M}, z_{M}\right)$ where $z_{M}=v t$. The spring force is given by $F_{\text {spring }}=k_{z}\left(z_{M}\right.$ $-z_{i}$ ) where $k_{z}$ is the spring constant of the cantilever. In this work, $k_{x}$ and $k_{y}$ are set to zero, i.e., the sideward (directions perpendicular to the pulling direction) motions of the biotin group in the binding pocket are not restrained, since such motions are essentially unconstrained in AFM unbinding experiments.

A tetramer structure ${ }^{21}$ of the streptavidin-biotin complex (Protein Data Bank code: 1swe) was used. The CHARMM package ${ }^{26}$ was used to add hydrogen atoms. The whole complex was then reoriented so that the possible unbinding pathway of biotin is parallel to the direction of the external force ( $z$ direction designated in this work). The potential parameters and charges of streptavidin are from the CHARMM force field for protein ${ }^{26}$ whereas the parameters and partial charges of biotin are the same as used by Izrailev et al. ${ }^{18}$ In AFM unbinding experiments, the biotin and the AFM tip are usually connected by a reactive bifunctional covalent crosslinker and should move together. To simplify, we let the position of the oxygen atom $\mathrm{O} 2$ of the biotin be exactly the same as the position of the AFM tip in our hybrid simulations as Grubmuller et al. ${ }^{17}$ did in their MD simulations. It has been $\operatorname{argued}^{27}$ that the binding pocket of avidin is inaccessible to water. Izrailev et al. ${ }^{18}$ attempted to place water molecules in the binding pocket and they found that the tight contact between biotin and its binding pocket makes it unlikely for more than one water molecule to fit inside the binding pocket. Hence we do not include explicit water molecules in this work. For the tetramer structure of the streptavidin-biotin complex, ${ }^{21}$ it has been found that the 3-4 loop (residues 45-52) of streptavidin adopts an open conformation for the unbound state and a closed conformation for the bound state. In AFM unbinding experiments, ${ }^{5-12}$ the biotin and the AFM tip are usually connected by a reactive bifunctional covalent cross-linker. Because of the existence of the cross-linker, the 3-4 loop of streptavidin may not close even after binding. Furthermore, during the experimental unbinding process, the 3-4 loop will adopt an open conformation. To facilitate the exit of biotin from the binding pocket, in MD simulations by Izrailev et al., ${ }^{18}$ the loop was preopened. Similarly, the open conformation of the 3-4 loop was adopted in this work.

For hybrid simulations, the equations of motion for the tip were integrated using a backward differential algorithm either over $200 \Delta t_{\text {tip }}$ or when the tip displacement during each loop of tip motion exceeds $0.2 \AA$. If the tip displacement reaches $0.2 \AA$ during the integration of tip motion equations, the hybrid simulation will return to MD relaxation. When stick-slip occurs, the tip will quickly slip from the binding pocket and the biotin moves a large distance even for a single step of cantilever motion. Thus, the criterion for a tip displacement of less than $0.2 \AA$ is critical; otherwise, the quasistatic assumption is violated. We have tried different maximum allowed tip displacements: $0.1,0.2,0.3$, and $0.4 \AA$. It has been found that for the case of $0.4 \AA$, occasionally, there are spikes for potential energy-time curves, which indicate the violation of quasistatic assumption. The value of $0.2 \AA$ for the maximum allowed tip displacement during the tip motion was used in the hybrid simulations, no spike was found from the potential energy evolution curves. The time step $\Delta t_{\text {tip }}$ used in this work is $0.25 \mu \mathrm{s}$ (equivalent to the displacement of the support by $0.0025 \AA$ at a pulling velocity of $1.0 \mu \mathrm{m} / \mathrm{s}$ ). Following the tip motion, the complex was relaxed by MD simulations with a time step $\Delta t_{\mathrm{MD}}$ of $1.0 \mathrm{fs}$. We found that MD relaxation over $300 \Delta t_{\text {tip }}, 600 \Delta t_{\text {tip }}$, and $1200 \Delta t_{\text {MD }}$ yielded the same force-distance curve, indicating that the relaxation of atomic system is quite fast. In this work, $400 \Delta t_{\mathrm{MD}}$ was used for MD relaxation. For MD simulations, initial velocities were assigned from a MaxwellBoltzmann distribution at $300 \mathrm{~K}$. The Berendsen method ${ }^{28}$ was used to keep the temperature of the system constant. The simulated system was coupled with a heat bath, using a coupling time of $0.1 \mathrm{ps}$. Bonds containing hydrogen are constrained by using the RATTLE method ${ }^{29}$ with a geometric tolerance of $10^{-6}$. Cell-linked list ${ }^{30}$ was employed to accelerate the simulations. The short-range nonbonded interactions were calculated by a switched potential with a switching function starting at $10 \AA$ and reaching zero at a distance of $11 \AA$. Electrostatic interactions were calculated by the shifted potential with a cutoff distance of $11 \AA$. For the streptavidin tetramer, only one pair of streptavidin-biotin was selected for unbinding. The center of mass of the streptavidin monomer to be unbound with the biotin group was fixed, which allowed the free rotational and internal motions of the system by external forces while the centers of mass of the other three streptavidin monomers were not fixed. With the alternative integration of the equations of motions for the tip and the atomic complex, the biotin was pulled out gradually. The hybrid simulations were performed up to a tip displacement of $15.0 \AA$. A graphical illustration of the hybrid simulation method is shown in Fig. 1. For clarity, only one pair of streptavidin-biotin was shown.

\section{RESULTS AND DISCUSSION}

In this work, the single-molecule interactions between streptavidin and biotin were investigated with the hybrid simulation method. The problems encountered in experiments, such as multiple-pair interactions, nonspecific interactions, and protein denaturization, can be avoided. To examine the loading-rate dependence of unbinding force found in AFM experiments, the unbinding of the streptavidin-biotin complex $^{21}$ was simulated with an AFM cantilever (spring constant $k_{z}=0.039 \mathrm{~N} / \mathrm{m}$ ) under different cantilever pulling velocities of $1.0,10$, and $50 \mu \mathrm{m} / \mathrm{s}$. This spring constant is in the range of spring constants for AFM cantilevers commonly used and the same as that used in AFM unbinding experiments by Lo et al. ${ }^{9}$ 


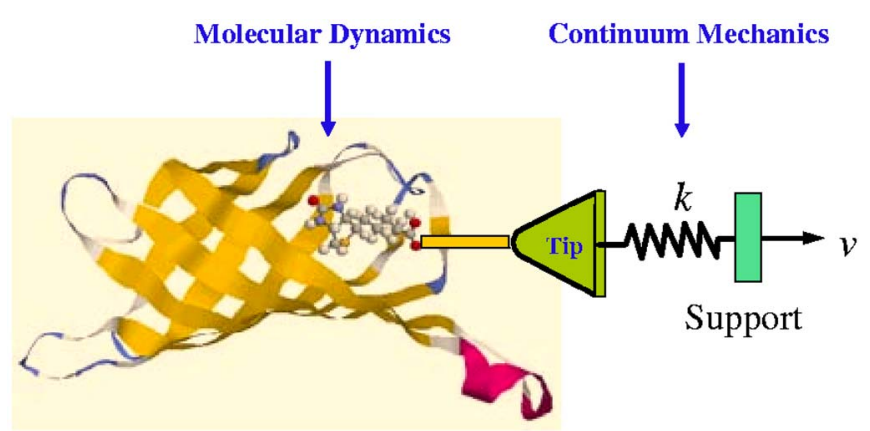

FIG. 1. (Color online) Illustration of the hybrid simulation method. The streptavidin is in ribbon representation while the biotin is in ball-stick representation. For clarity, only one pair of streptavidin-biotin was shown.

\section{A. Force-distance relationship}

The simulated force-distance (or time) curves at pulling velocities of $1.0,10$, and $50 \mu \mathrm{m} / \mathrm{s}$ are shown in Figs. 2(a)-2(c), respectively. As seen from these figures, the external spring forces increase almost linearly before reaching the maximum and then drop sharply. This typical stick-slip behavior of force-distance curves observed in AFM experiments is well reproduced by the hybrid simulation method. The simulated unbinding (or rupture) force is defined as the largest spring force experienced during the unbinding simulation process. At pulling velocities of 1.0, 10, and $50 \mu \mathrm{m} / \mathrm{s}$, the unbinding forces of the streptavidin-biotin complex are 227,349 , and $478 \mathrm{pN}$, respectively, whereas the rupture times of the complex upon AFM pulling are 5.95, 0.94, and $0.257 \mathrm{~ms}$, respectively. Thus, the time scale gap between AFM experiments and conventional MD simulations is bridged by the hybrid simulation method.

\section{B. Cantilever position versus tip position}

The cantilever position versus tip position curves of the unbinding process are shown in Figs. 3(a)-3(c) for different pulling velocities. These figures indicate the unbinding pathway of the streptavidin-biotin complex. This information cannot be readily extracted from experiments, since only the position of the support is known while one cannot locate the position of the AFM tip from unbinding experiments. At the pulling velocity of $1.0 \mu \mathrm{m} / \mathrm{s}$, as shown in Fig. 3(a), the biotin moves away from its initial position and fluctuates around the position of $0.3 \AA$ (the first local barrier). Later, the biotin jumps to a new position of $1.0 \AA$, which means that the biotin overcomes the first energy barrier, and moves to a new barrier at the position of $1.0 \AA$. With the further increase of the external force, the biotin overcomes this new barrier and reaches another barrier at $2.6 \AA$, then the biotin slips out of the streptavidin binding pocket quickly, which is reflected by the steep increase of tip distance. This steep increase of tip distance in Fig. 3(a) corresponds to the sudden

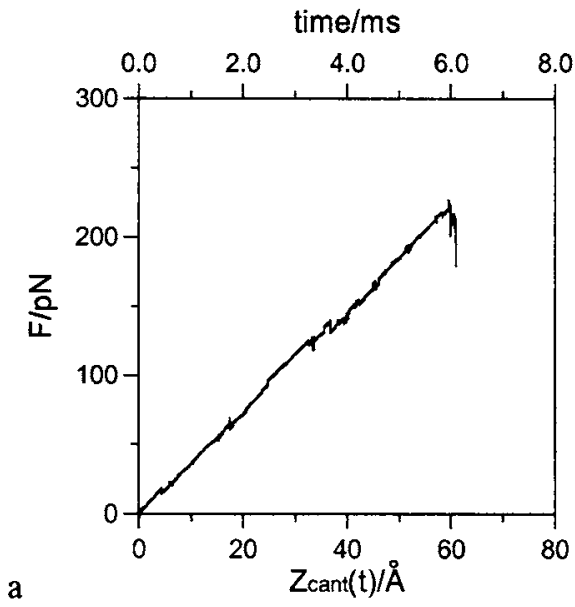

a

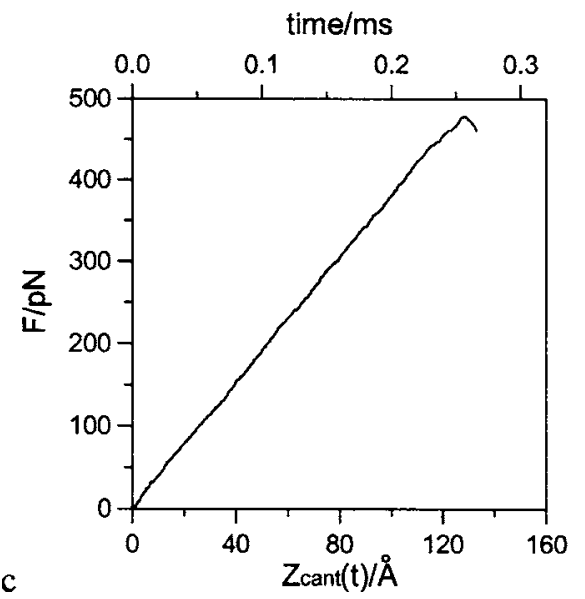

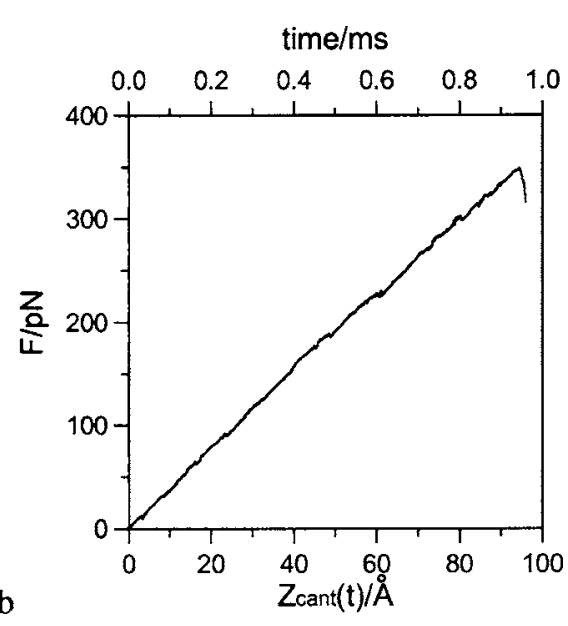

b
FIG. 2. Force-distance (or time) curves for the unbinding of a streptavidin-biotin complex with a cantilever of $k_{z}=0.039 \mathrm{~N} / \mathrm{m}$ at the pulling velocities of $1.0 \mu \mathrm{m} / \mathrm{s}$ (a), $10 \mu \mathrm{m} / \mathrm{s}$ (b), and $50 \mu \mathrm{m} / \mathrm{s}$ (c) from hybrid simulations. 

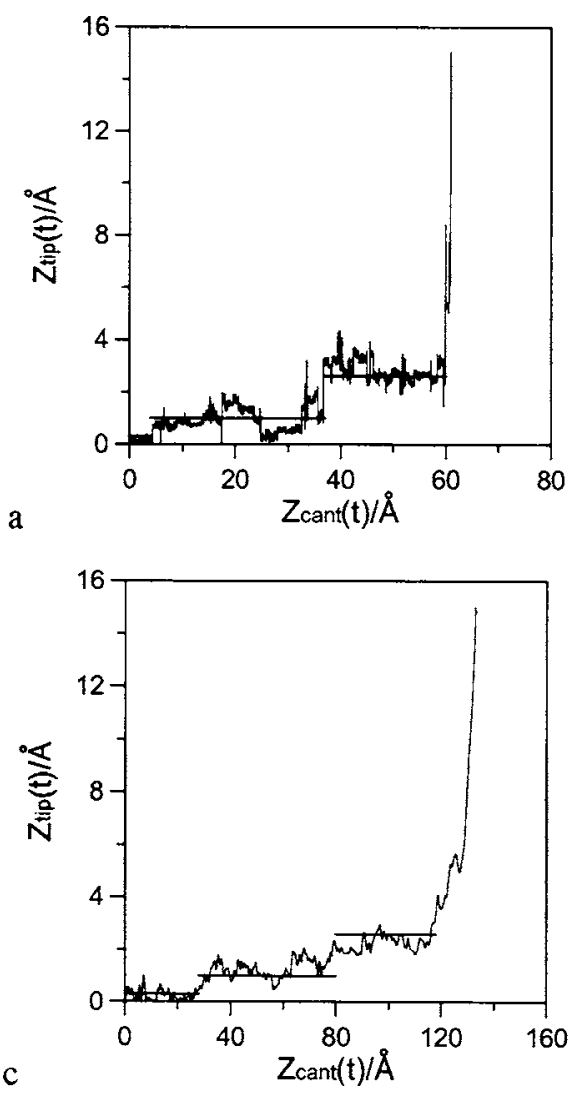

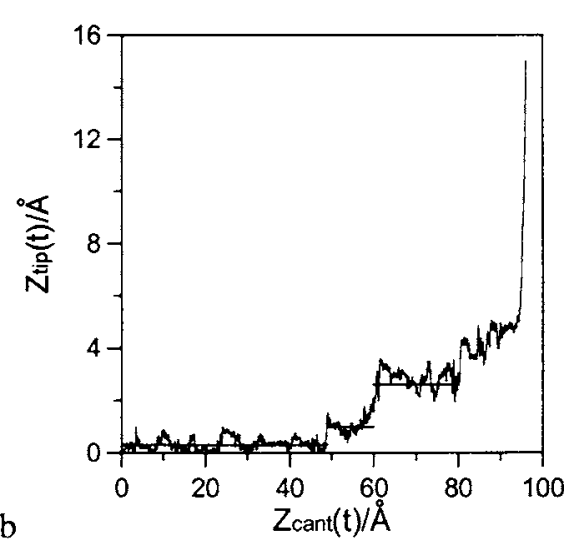

b

FIG. 3. Cantilever position vs tip position curves for the unbinding of a streptavidin-biotin complex with a cantilever of $k_{z}=0.039 \mathrm{~N} / \mathrm{m}$ at the pulling velocities of $1.0 \mu \mathrm{m} / \mathrm{s}$ (a), $10 \mu \mathrm{m} / \mathrm{s}$ (b), and $50 \mu \mathrm{m} / \mathrm{s}$ (c) from hybrid simulations. The horizontal bars indicate the locations of energy barriers. decrease of force in Fig. 2(a). At the pulling velocities of 10 and $50 \mu \mathrm{m} / \mathrm{s}$, the simulated cantilever position versus tip position curves exhibit similar behaviors as those at the pulling velocity of $1.0 \mu \mathrm{m} / \mathrm{s}$.

\section{Unbinding pathway}

From the ribbon representation structure of streptavidin in Fig. 1, it can be seen that streptavidin is organized as eight-stranded, sequentially connected, antiparallel $\beta$ sheets. The sheets are formed of coiled polypeptide chains with a staggered pattern of adjacent strand hydrogen-bond registration. The biotin binds in pockets at the end of streptavidin $\beta$ barrels. The residues lining the pockets are primarily polar amino acids. $^{21}$

Figures 4(a)-4(e) show a few typical configurations found during the unbinding process at the pulling velocity of $1.0 \mu \mathrm{m} / \mathrm{s}$. For the initial configuration in Fig. 4(a), the hydrogen bonds are distributed in three areas: the inner, the middle, and the outer of the binding pocket. In the inner part, an extensive pattern of five hydrogen bonds with the ureido group of biotin are found, i.e., the Ser45 OH with biotin N1, Asp128 OD2 with biotin N2, and Tyr43 OH, Asn23 NH, Ser27 $\mathrm{OH}$ with the biotin ureido oxygen. In the middle part, the biotin sulfur interacts with the $\mathrm{OH}$ of Thr90. In the outer part, the two valeryl oxygen atoms of biotin are hydrogen bonded to the backbone NH of Asn49 and the OH of Ser88. Under the force exerted by the support, the biotin moves from its original binding position gradually. When the tip moves $0.3 \AA$ from its original position, two hydrogen bonds break first, i.e., the sulfur atom of biotin with the $\mathrm{OH}$ of Thr90 and one valeryl oxygen with the backbone $\mathrm{NH}$ of
Asn49. This is shown in Fig. 4(b). Figure 4(c) displays the configuration corresponding to the location of energy barrier at $1.0 \AA$. It can be seen that another two hydrogen bonds break, i.e., the Ser45 OH with biotin N1 and the Tyr43 OH with the biotin ureido oxygen. In Fig. 4(d), for the location of energy barrier at $2.6 \AA$, the biotin sulfur interacts with the $\mathrm{OH}$ of Thr90 and Ser45 $\mathrm{OH}$ interacts with the biotin ureido oxygen while the hydrogen bond interaction between Asn23 $\mathrm{NH}$ and the biotin ureido oxygen breaks. After this major

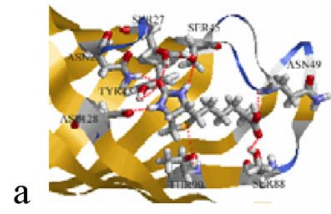

b
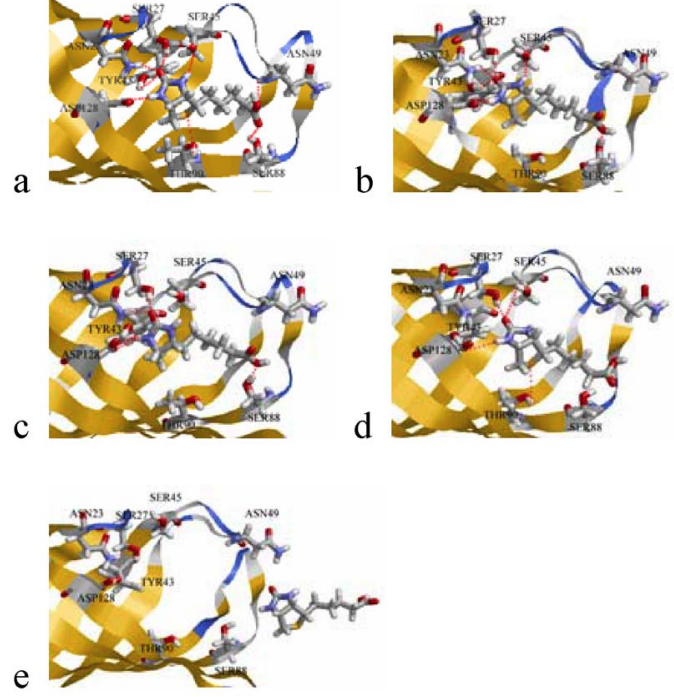

FIG. 4. (Color online) Snapshots of the streptavidin-biotin binding pocket during an unbinding process at the initial stage (a), at $0.3 \AA$ (b), at $1.0 \AA$ (c), at $2.6 \AA$ (d), and at the final unbinding stage (e) from hybrid simulations with a cantilever of $k_{z}=0.039 \mathrm{~N} / \mathrm{m}$ at the pulling velocity of $1.0 \mu \mathrm{m} / \mathrm{s}$. The dashed lines indicate hydrogen bonds. 


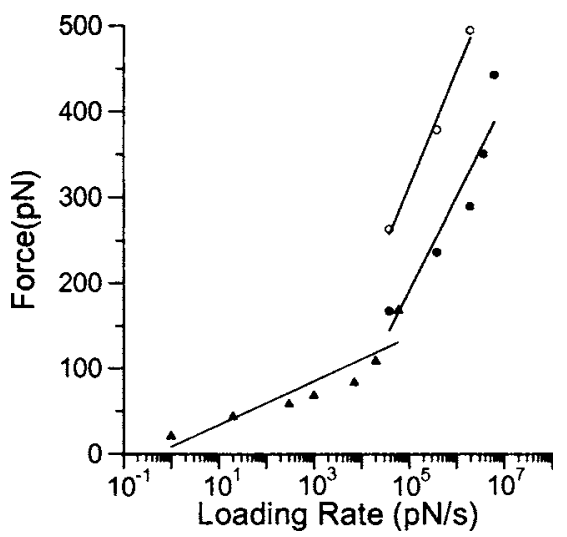

FIG. 5. Loading rate dependence of unbinding forces for a cantilever of $k_{z}=0.039 \mathrm{~N} / \mathrm{m}$. Open circles are for the hybrid simulation results. Solid circles are for the AFM data (Ref. 9). Triangles are for the BFP data (Ref. 13). The lines are drawn to guide eyes.

barrier, the biotin leaves out of the binding pocket quickly. The final unbinding configuration is shown in Fig. 4(e). In general, the unbinding of the streptavidin-biotin complex involves the breakage and formation of multiple hydrogen bonds between the ureido group and valeryl oxygen of biotin and the binding site residues of streptavidin.

\section{Loading-rate dependence of unbinding forces}

To get good statistics of the unbinding forces, we performed eight hybrid simulations under each condition from different initial configurations (results not shown). Under each simulation condition (same cantilever spring constant and same pulling velocity), the shapes of the obtained forcedistance curves and the tip position versus cantilever position curves are very similar to those in Figs. 2(a)-2(c) and Figs. 3(a)-3(c), respectively. Major locations of energy barriers around $0.3,1.0$, and $2.6 \AA$ are found in all cases. The average unbinding forces are $263 \pm 36,378 \pm 42$, and $494 \pm 39 \mathrm{pN}$ at pulling velocities of $1.0,10$, and $50 \mu \mathrm{m} / \mathrm{s}$, respectively. Experimentally, it has been observed ${ }^{5-12}$ that there is a distribution of determined unbinding forces at each condition.

One advantage of simulation studies is that each parameter can be studied separately under well-controlled conditions. In AFM pulling experiments, loading rate is often used, which is the product of cantilever spring constant and pulling velocity. Previous AFM experiments show the logarithmic relationship between unbinding force and loading rate. The average unbinding forces by hybrid simulations of a cantilever (spring constant of $0.039 \mathrm{~N} / \mathrm{m}$ ) at different cantilever pulling velocities are shown in Fig. 5 together with experimental results by AFM (Ref. 9) and BFP. ${ }^{13}$ The hybrid simulation results show a similar logarithmic trend to the experimental data. Thus, the loading-rate dependence of unbinding forces predicted by theory ${ }^{16}$ and found by experiments ${ }^{9,12,13}$ is also observed from the hybrid simulations.

\section{CONCLUSIONS}

In this work, a hybrid molecular simulation method, which combines continuum mechanics and molecular dynamics, was used to study the single-molecule unbinding force of a streptavidin-biotin complex at the time scale of AFM unbinding experiments. For the cantilever with a spring constant similar to that used in AFM experiments, the logarithmic relationship between unbinding forces of the streptavidin-biotin complex and loading rates was observed. The simulated force-distance curves display a typical stickslip behavior as in AFM experiments. The three major locations of energy barriers of the streptavidin-biotin complex around $0.3,1.0$, and $2.6 \AA$ along the unbinding pathway were found. The streptavidin-biotin unbinding involves the breakage and formation of multiple hydrogen bond interactions between the ureido group and valeryl oxygen of biotin and the binding site residues of streptavidin. Our hybrid simulations cannot only interpret unbinding AFM experiments but also provide detailed molecular information not available in AFM experiments.

\section{ACKNOWLEDGMENT}

This work was supported by the National Science Foundation (CMS-0302139).

${ }^{1}$ E. Evans, Annu. Rev. Biophys. Biomol. Struct. 30, 105 (2001).

${ }^{2}$ M. Horton, G. Charras, and P. Lehenkari, J. Recept. Signal Transduct. Res. 22, 169 (2002).

${ }^{3}$ R. Lavery, A. Lebrun, J. F. Allemand, D. Bensimon, and V. Croquette, J. Phys.: Condens. Matter 14, R383 (2002).

${ }^{4}$ J. W. Weisel, H. Shuman, and R. I. Litvinov, Curr. Opin. Struct. Biol. 13, 227 (2003).

${ }^{5}$ G. U. Lee, D. A. Kidwell, and R. J. Colton, Langmuir 10, 354 (1994).

${ }^{6}$ E. L. Florin, V. T. Moy, and H. E. Gaub, Science 264, 415 (1994).

${ }^{7}$ V. T. Moy, E. L. Florin, and H. E. Gaub, Science 266, 257 (1994).

${ }^{8}$ Y. S. Lo, N. D. Huefner, W. S. Chan, F. Stevens, J. M. Harris, and T. P. Beebe, Langmuir 15, 1373 (1999).

${ }^{9}$ Y. S. Lo, Y. J. Zhu, and T. P. Beebe, Langmuir 17, 3741 (2001).

${ }^{10}$ Y. S. Lo, J. Simons, and T. P. Beebe, J. Phys. Chem. B 106, 9847 (2002).

${ }^{11}$ J. Wong, A. Chilkoti, and V. T. Moy, Biomol. Eng. 16, 45 (1999).

${ }^{12}$ C. Yuan, A. Chen, P. Kolb, and V. T. Moy, Biochemistry 39, 10219 (2000).

${ }^{13}$ R. Merkel, P. Nassoy, A. Leung, K. Ritchie, and E. Evans, Nature (London) 397, 50 (1999).

${ }^{14}$ K. Svoboda and S. M. Block, Annu. Rev. Biophys. Biomol. Struct. 23, 247 (1994).

${ }^{15}$ A. B. Patel, S. Allen, M. C. Davies, C. J. Roberts, S. J. B. Tendler, and P. S. Williams, J. Am. Chem. Soc. 126, 1318 (2004).

${ }^{16}$ E. Evans and K. Ritchie, Biophys. J. 72, 1541 (1997).

${ }^{17}$ H. Grubmuller, B. Heymann, and P. Tavan, Science 271, 997 (1996).

${ }^{18}$ S. Izrailev, S. Stepaniants, M. Balsera, Y. Oono, and K. Schulten, Biophys. J. 72, 1568 (1997).

${ }^{19}$ B. Heymann and H. Grubmuller, Chem. Phys. Lett. 303, 1 (1999).

${ }^{20}$ E. Galligan, C. J. Roberts, M. C. Davies, S. J. B. Tendler, and P. M. Williams, J. Chem. Phys. 114, 3208 (2001).

${ }^{21}$ S. Freitag, I. LeTrong, L. Klumb, P. S. Stayton, and R. E. Stenkamp, Protein Sci. 6, 1157 (1997).

${ }^{22}$ Y. S. Leng and S. Y. Jiang, J. Chem. Phys. 113, 8800 (2000).

${ }^{23}$ Y. S. Leng and S. Y. Jiang, J. Am. Chem. Soc. 124, 11764 (2002).

${ }^{24}$ L. Z. Zhang, Y. S. Leng, and S. Y. Jiang, Langmuir 19, 9742 (2003).

${ }^{25}$ L. Y. Li, Q. M. Yu, and S. Y. Jiang, J. Phys. Chem. B 103, 8290 (1999).

${ }^{26}$ A. D. MacKerell, Jr., D. Bashford, R. L. Bellott et al., J. Phys. Chem. B 
102, 3586 (1998)

${ }^{27}$ L. Pugliese, A. Coda, M. Malcovati, and M. Bolognesi, J. Mol. Biol. 231, 698 (1993).

${ }^{28}$ H. J. C. Berendsen, J. P. M. Postma, W. F. van Gunsteren, A. Dinola, and
J. R. Haak, J. Chem. Phys. 81, 3684 (1984).

${ }^{29}$ H. C. Andersen, J. Comput. Phys. 52, 24 (1983).

${ }^{30}$ D. J. Auerbach, W. Paul, C. Lutz, A. F. Bakker, W. E. Rudge, and F. F. Abraham, J. Phys. Chem. 91, 4881 (1987). 